\title{
Haploinsufficiency of RanBP2 is neuroprotective against light-elicited and age-dependent degeneration of photoreceptor neurons
}

\author{
K-in Cho ${ }^{1,3}, \mathrm{H} \mathrm{Yi}^{1{ }^{1,3}}$, A Yeh ${ }^{1}$, N Tserentsoodol ${ }^{1}$, L Cuadrado ${ }^{1}, \mathrm{~K}$ Searle ${ }^{1}$, Y Hao ${ }^{1}$ and PA Ferreira ${ }^{*, 1,2}$
}

Prolonged light exposure is a determinant factor in inducing neurodegeneration of photoreceptors by apoptosis. Yet, the molecular bases of the pathways and components triggering this cell death event are elusive. Here, we reveal a prominent agedependent increase in the susceptibility of photoreceptor neurons to undergo apoptosis under light in a mouse model. This is accompanied by light-induced subcellular changes of photoreceptors, such as dilation of the disks at the tip of the outer segments, prominent vesiculation of nascent disks, and autophagy of mitochondria into large multilamellar bodies. Notably, haploinsufficiency of Ran-binding protein-2 (RanBP2) suppresses apoptosis and most facets of membrane dysgenesis observed with age upon light-elicited stress. RanBP2 haploinsufficiency promotes decreased levels of free fatty acids in the retina independent of light exposure and turns the mice refractory to weight gain on a high-fat diet, whereas light promotes an increase in hydrogen peroxide regardless of the genotype. These studies demonstrate the presence of age-dependent and RanBP2mediated pathways modulating membrane biogenesis of the outer segments and light-elicited neurodegeneration of photoreceptors. Furthermore, the findings support a mechanism whereby the RanBP2-dependent production of free fatty acids, metabolites thereof or the modulation of a cofactor dependent on any of these, promote apoptosis of photoreceptors in concert with the light-stimulated production of reactive oxygen species.

Cell Death and Differentiation (2009) 16, 287-297; doi:10.1038/cdd.2008.153; published online 24 October 2008

The retina comprises a well-defined neurocircuitry mediating the capture, processing, and transmission of photon stimuli to high-order processing centers in the brain. The primary neurons of the retina, rod and cone photoreceptors, mediate the physicochemical transduction of light. Although several components of the light transduction cascade promote the degeneration of photoreceptors upon inherited mutations in the cognate genes, ${ }^{1}$ light also acts as a powerful inducer of degeneration of these neurons in wild-type mouse strains. ${ }^{2}$ Neurodegeneration elicited by light and age appears to vary in multiple genetic backgrounds, thus supporting the presence of various genetic modifiers of cell death upon selective stressors. $^{3-5}$

To date, few loci conferring resistance to light damage have been identified in genetically altered mice. These include mice lacking the expression of Rpe65, Rho, and mice harboring the Rpe65 Leu450Met mutation. ${ }^{5-9}$ Although some of these loci appear to have no impact on age-related retinal degeneration, quantitative trait loci have been implicated in age-related retinal degeneration, but the identities of the genes implicated in this process remain elusive. ${ }^{3,4}$ Regardless, cumulative damage from oxidative stress appears to play a determinant role in the development of age-related phenotypes of photoreceptors in part as the result of marked and uneven oxygen tension and metabolic demands, across the retina, that make photoreceptors particularly vulnerable to oxidative damage. ${ }^{10-13}$ To this effect, overexpression of erythropoietin in transgenic mice is neuroprotective against light-induced but not inherited retinal degeneration. ${ }^{14}$ Hence, the data hint of a link between light- and age-dependent death of photoreceptor neurons. On the other hand, the phenotypic analyses of genetically engineered mouse models support that lightinduced degeneration is independent of the activation of phototransduction, but dependent on the light-receptor, rhodopsin, and that independent and poorly defined mechanisms triggering apoptosis may operate for light-induced, age-dependent, and inherited forms of retinal degeneration. ${ }^{5,8}$ In addition, light-elicited degeneration of photoreceptors may act synergistically with certain forms of inherited degeneration selectively affecting these neurons, because neurodegeneration is exacerbated by light in certain mouse models with inherited degeneration of photoreceptors. ${ }^{15-19}$

\footnotetext{
${ }^{1}$ Department of Ophthalmology, Duke University Medical Center, Durham, NC, USA and ${ }^{2}$ Department of Molecular Genetics and Microbiology, Duke University Medical Center, Durham, NC, USA

*Corresponding author: PA Ferreira, Department of Ophthalmology, Molecular Genetics and Microbiology, Duke University Medical Center, DUEC 3802, Erwin Road, Durham, NC 27710, USA. Tel: + 919684 8457; Fax: + 919684 6545; E-mail: ferre044 @mc.duke.edu

${ }^{3}$ These authors contributed equally to this work

Keywords: Ran-binding protein 2 (RanBP2); neuroprotection; light; free fatty acids; photoreceptor neurons; apoptosis

Abbreviations: DAG, diacylglycerol; DAG, diacylglycerol; DHA, docosahexenoic acid; FFAs, free fatty acids; $\mathrm{H}_{2} \mathrm{O}_{2}$, hydrogen peroxide; LDs, lipid droplets; MLBs, multilamellar bodies; ONL, outer nuclear layer (nuclei of photoreceptors); PIP $_{2}$, phosphatidylinositol 4,5-biphosphate; PI-PLC- $\beta 4$, phosphatidylinositol-phospholipase C$\beta 4$; RanBP2, Ran-binding protein 2; RIS, rod inner segment (compartment) of photoreceptors; ROS, rod outer segment (compartment) of photoreceptors; RPE, retinal pigment epithelium; RPE65, retinal pigment epithelium-specific protein $65 \mathrm{kDa}$; SUMO-1, small ubiquitin-like modifier-1; TEM, transmission electron microscope; Topo Il $\alpha$, topoisomerase-Il $\alpha$; TUNEL, TdT-mediated dUTP nick-end labeling
}

Received 05.5.08; revised 08.9.08; accepted 19.9.08; Edited by D Kaplan; published online 24.10.08 
Hence, the identification of novel components modulating the death of photoreceptors upon light and aging are likely to provide critical insights to novel pathways underlying the molecular bases of neurodegeneration upon various stress stimuli.

The Ran-binding protein-2 (RanBP2) is at the nexus of multiple subcellular and molecular processes underlying nuclear-cytoplasmic trafficking, ${ }^{20-22}$ transport and function of mitochondria, ${ }^{23}$ modulation of proteasome function and protein homeostasis, ${ }^{24-26}$ and the modulation of proteinprotein interaction by sumoylation in cultured cells. ${ }^{27-30}$ Notably, haploinsufficiency of RanBP2 in the mouse in combination with diet and genetic background triggers defined age-related phenotypes manifested by perturbation of growth and glucose catabolism. ${ }^{31}$ In addition, further decrease of the levels of RanBP2 in another mouse model harboring a hypomorphic allele of RanBP2 in a mixed genetic background promotes missegregation of chromosomes (aneuploidy) in mitotic cells and carcinogen-elicited and age-dependent tumorigenesis without overall impairment of nuclear-cytoplasmic trafficking, mitotic spindle formation, and protein SUMO modification. ${ }^{32}$ Here, we reveal that light-induced susceptibility to damage and apoptosis of photoreceptor neurons increases prominently between 12- and 24-week-old inbred 129P2/OlaHsd mice, and that haploinsufficiency of RanBP2 in these mice suppresses strongly the age- and light-dependent increase of damage and cell death of photoreceptors. Moreover, the neuroprotective effects caused by a deficit in RanBP2 is reflected by a significant decrease of free fatty acids (FFAs), which upon light-induced oxidative stress, may suppress apoptosis and preceding phenotypes, such as membrane dysgenesis.

\section{Results}

Light-induced morphological changes of photoreceptor neurons by RanBP2 haploinsufficiency and age. Earlier, we have identified phenotypes in RanBP2 haploinsufficient mice that are manifested in an age-dependent fashion. ${ }^{31}$ In light of the role of RanBP2 in retinal function ${ }^{31}$ and multiple processes that may contribute to the development of manifestations linked to cell death events, we assessed the role of RanBP2 in the development of light- and age-dependent phenotypes linked to the damage and death of photoreceptor neurons. Because age-dependent manifestations were observed between 12- and 24-week-old RanBP2 ${ }^{+/+}$and RanBP2 $^{+/-}$ mice on an inbred 129P2/OlaHsd background, ${ }^{31}$ we examined the effect of constant white illumination (1200 lux) for a period of $48 \mathrm{~h}$ on inducing gross morphological abnormalities of retinal neurons, in particular photoreceptors, between 12- and 24week-old RanBP2 $2^{+/+}$and RanBP2 ${ }^{+/-}$mice on the same genetic background. We found that 12 -week-old RanBP2 ${ }^{+/}$ mice presented vacuolization of the outer segment compartment of photoreceptors that was limited to the distal (upper) portion of this compartment. This damage was less apparent in RanBP2 $2^{+/+}$mice (Figure $1 \mathrm{a}$ and b). In addition, few scattered and pyknotic nuclei were visible in both RanBP2 $^{+/+}$and RanBP2 ${ }^{+/-}$mice, but no significant changes were observed in the organization of the outer nuclear layer and inner segment compartment of photoreceptors. In contrast, the central regions of the retina of 24-week-old RanBP2 $2^{+/+}$mice presented drastic morphological changes of the outer and inner segment compartments of photoreceptors, including condensed inner segments, overall disorganization of outer segments and outer nuclear layer, the presence of cystic spaces and cellular-like debris in the inner segments, and a strong increase of widespread condensed nuclei of photoreceptors that led to a noticeable decrease of the thickness of the outer nuclear layer (rows of nuclei; Figure 1c, Supplementary Figure 1). These phenotypic abnormalities in the central regions of the retina were largely alleviated in $\mathrm{RanBP}^{+/-}$mice (Figure 1d) and peripheral regions of the retina of both genotypes (Figure 1e and f).

Haploinsufficiency of RanBP2 alleviates membrane dysgenesis in photoreceptors triggered by prolonged light exposure. We assessed further the abnormalities of photoreceptors upon light-induced degeneration at ultrastructural level. There was a strong increase in the overall disorganization of the rod and inner segment subcellular compartments of photoreceptors in 24-week-old RanBP2 $^{+/+}$mice compared with 12-week-old RanBP2 $2^{+/+}$ mice (Figure $2 a$ and $b$ ). This increase in disorganization was largely suppressed in RanBP2 ${ }^{+/-}$mice (Figure $2 \mathrm{c}$ and d). The subcellular changes in photoreceptors induced by light comprised a substantial increase of dilated disks at the tip of the outer segments, the presence of condensed (electrodense) inner segments (star, Figure 2b), large cystic spaces between outer segments and between nuclei (arrowheads, Figure 2b), vacuoles in the inner segments, and migration of mitochondria to the outer nuclear layer. These age-dependent pathological phenotypes were also lessened in RanBP2 ${ }^{+/-}$mice (Figure $2 \mathrm{c}$ and d). In addition, there are at least two unique features at ultrastructural level that were observed in photoreceptors of 24-week-old $\operatorname{RanBP}^{+/+}$, but not RanBP2 $2^{+/-}$mice. First, RanBP2 $2^{+/+}$ mice presented a prominent accumulation of multivesicular bodies at the base of the outer segments (Figure $2 b$ and e) that disrupted the stacking of the nascent disks of the outer segments. This was accompanied often by the fragmentation of the nascent disks, formation of large lateral disk lamellas, and numerous vesicular bodies of various sizes (Figure $2 \mathrm{e}$, inset picture). Conversely, RanBP2 ${ }^{+/}$mice appear largely to present normal formation of nascent disks, but two large vesicular bodies were often visible between nascent disks causing the focal disruption of their stacking (Figure $2 f$, arrows), and small crescent vesicular bodies appear to bulge also from the rims of some nascent disks (Figure 2f, arrowheads). In both genotypes, such vesicular bodies seem comprised of a single membrane leaflet or no membrane could be discerned. Second, the inner segment compartment often presented large multilamellar bodies (MLBs) with loose lamellae of variable interlamellar space surrounding fragmented mitochondria, possibly indicating the degeneration of mitochondria into MLBs (Figure 2e and g). This phenotype was not observed in RanBP2 ${ }^{+/-}$mice. Mitochondria also presented the dilation and fragmentation of the cristae. Thus, the data support the existence of a RanBP2-dependent switch rendering photoreceptors susceptible to damage by light with age and of a 

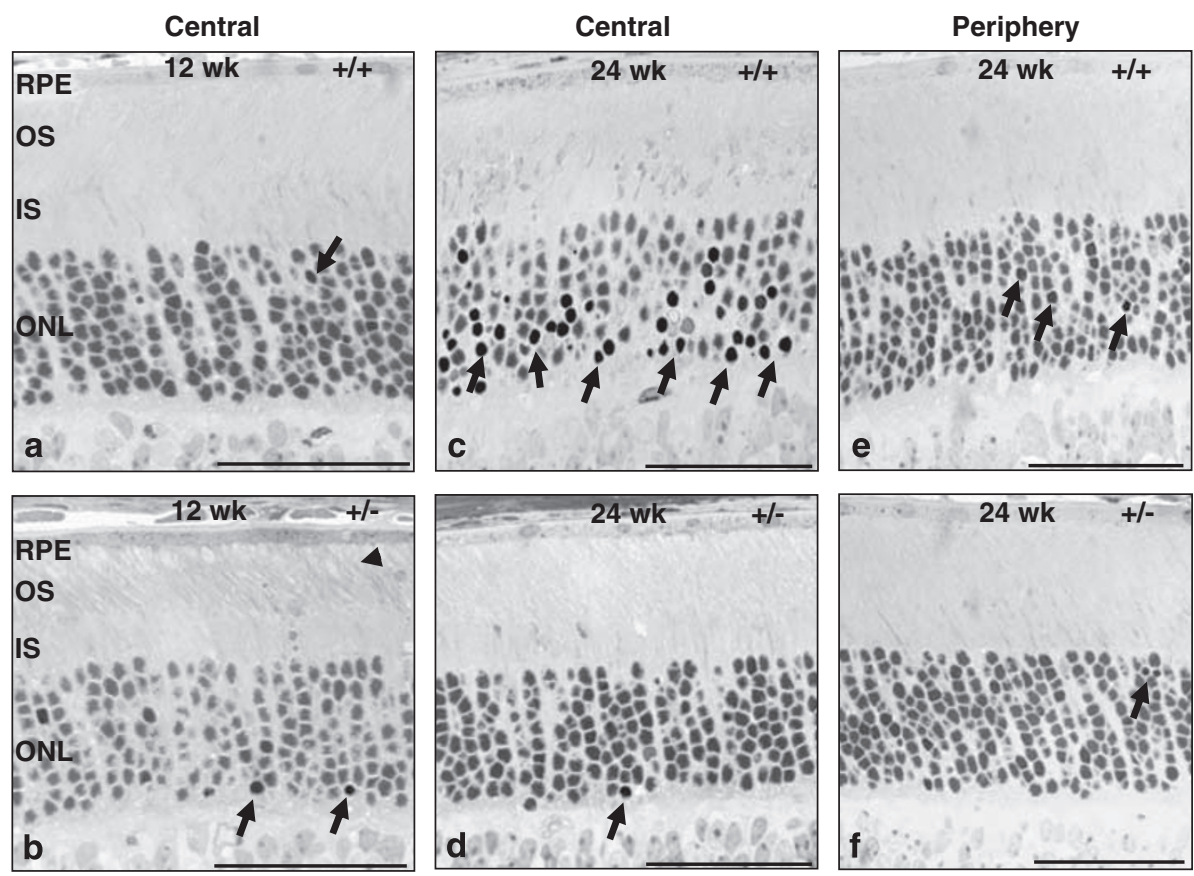

Figure 1 Age-dependent light damage of photoreceptors is strongly reduced in RanBP2 haploinsufficient mice. Light photomicrographs of methylene blue-stained sections of central (a-d) and peripheral (e and $\mathbf{f})$ regions of the retina of 12- (a and $\mathbf{b})$ and 24 -week-old (c-f) $R a n B P 2^{+/+}(\mathbf{a}, \mathbf{c}$, and $\mathbf{e})$ and $R a n B P 2^{+/-}$mice (b, $\mathbf{d}$, and $\left.\mathbf{f}\right)$. There is a strong increase in pyknotic nuclei in 24-week-old wild-type mice compared with 12-week-old wild-type mice (arrows pointing to intense nuclei staining) in the central retina ( $\mathbf{a}$ and $\mathbf{c}$ ) that is accompanied by the disorganization of the outer nuclear layer. The periphery of the retina is spared largely from pyknosis (e). In contrast, no apparent differences in pyknosis are observed between 12- and 24-week-old RanBP2 ${ }^{+/-}$mice (b, d, and f), but some vacuolization of the tip of the outer segments can be noted (arrowhead). RPE, retina pigment epithelium; OS, outer segment of rod photoreceptors; IS, inner segment of rod photoreceptors; ONL, outer nuclear layer (nuclei of photoreceptors). Scale bar, $40 \mu \mathrm{m}$. A colour version of this figure is available online

neuroprotective mechanism by RanBP2 that halts the agedependent effect of light degeneration. Interestingly, all mice exhibited the dilation and disruption of the disks at the tip of the outer segments of photoreceptors independently of their age and genotype (Figure $2 a-d)$. This phenotype was not observed in RanBP2 $^{+/+}$mice reared under low ( $<70$ lux) illumination (Supplementary Figure 2). Hence, there is a nonselective RanBP2-independent effect of light in the development of dilated and disorganized disks at the tip of photoreceptors that is common at the early stages of lightinduced damage of photoreceptors.

Differences in the light-induced apoptosis of photoreceptors across the retina caused by aging and RanBP2 haploinsufficiency. We performed morphometric analyses on the impact of light on eliciting apoptosis of photoreceptor neurons in 12- and 24-week old RanBP2 ${ }^{+/+}$ and RanBP2 $^{+/-}$mice. We surveyed retina sections for the presence of DNA fragmentation in nuclei by TdT-mediated dUTP nick-end labeling (TUNEL) assay. Among retinal neurons, light-elicited apoptosis was restricted to nuclei of photoreceptor neurons regardless of the age and genotype of the mice (Figure 3). There was no apparent difference in cell death between 12-week-old RanBP2 ${ }^{+/+}$and RanBP2 $^{+/-}$ mice (Figure 3a and b). Conversely, 24-week-old RanBP2 ${ }^{+/+}$ and $R_{\text {anBP2 }}{ }^{+/}$mice presented a substantial difference in the number of apoptotic nuclei in photoreceptors (Figure 3c-f).
To this effect, wild-type mice exhibited a strong increase in the apoptosis of photoreceptors (Figure $3 c$ and e) in comparison to age-matched $\operatorname{RanBP2} 2^{+/-}$mice (Figure $3 d$ and f). The same was also observed between 12- and 24-week-old wild-type mice (Figure $3 a, c$ and e), but not between 12- and 24-week-old RanBP2 ${ }^{+/-}$mice (Figure 3b, d and f). These observations were confirmed by quantitative analyses of TUNEL-positive nuclei of photoreceptors derived from several sections of the eyecup. A fivefold significant increase of the total number of apoptotic photoreceptors was observed in 24-week-old RanBP2 $2^{+/+}$mice compared with 12-week-old RanBP2 ${ }^{+/+}$mice (Figure $4 a$ ), whereas there was no significant difference between 12- and 24-week-old RanBP2 $^{+/-}$mice (Figure 4b). At the age of 12 weeks, both wild-type and RanBP2 $^{+/-}$mice had similar total number of apoptotic cells (Figure 4c). However, RanBP2 $2^{+/}$mice exhibited significantly less apoptotic photoreceptors compared with $R a n B P 2^{+/+}$mice at the age of 24 weeks (Figure 4d). Hence, there is an age-dependent increase in the susceptibility of photoreceptors to undergo cell death upon light-elicited stress that is suppressed by haploinsufficiency of RanBP2.

In addition, quantitative morphometric analysis was performed on multiple central and peripheral sections of retinas to probe for potential regional differences of susceptibility to apoptosis of photoreceptors. The amount of cell death was normalized against the area of outer nuclear region analyzed. 
290
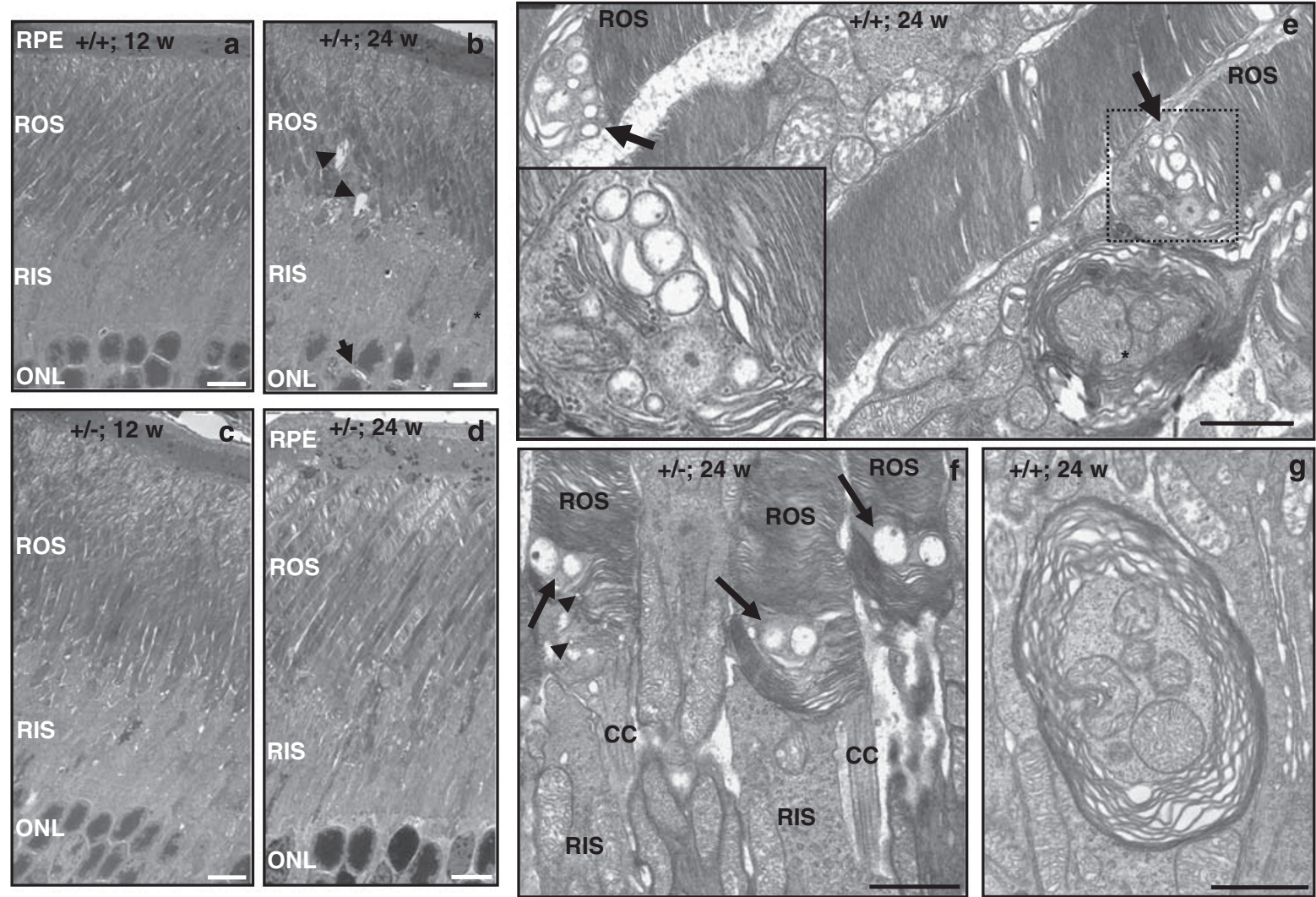

Figure 2 Electron micrographs depicting the age-dependent ultrastructural changes of inner and outer segments of photoreceptors of 12-and 24-week-old RanBP2 ${ }^{+/+}$ and $\mathrm{RanBP}^{+/-}$mice upon prolonged light exposure. There is an overall increase in the subcellular disorganization of morphological features of the inner and outer segments of photoreceptors in 24-week-old RanBP2 ${ }^{+/+}$mice (b) compared with 12-week-old RanBP2 ${ }^{+/-}$mice (a). This is reflected by an increase of cystoid spaces between nuclei (arrow) and outer segments (arrowheads), shrinking, and condensed inner segments (star), formation of vacuoles in the inner segment, and dilation of the disks at the tip of the outer segments. The development of these subcellular pathologies, except for the dilation of the disks, was strongly decreased in 24-week-old RanBP2 ${ }^{+/-}$mice (d) compared with 12-week-old RanBP2 ${ }^{+-}$mice (c). $\mathbf{e}, \mathbf{f}$, and $\mathbf{g}$ depict high magnification images of single membrane multivesicular bodies at the base of the outer segments (e), large vesicular body duets (arrows), and crescent vesicles in nascent disk rims (arrowheads) at the base of the outer segments of RanBP2 ${ }^{+/-}$mice (f), and multilamellar bodies engulfing mitochondria in RanBP2 $2^{+/}$mice (e, star; g). RPE, retina pigment epithelium; ROS, rod outer segment of photoreceptors; RIS, rod inner segment of photoreceptors; ONL, outer nuclear layer (nuclei of photoreceptors); CC, connecting cilium. Scale bar in a-d, $6 \mu \mathrm{m}$; scale bar in $\mathbf{e}-\mathbf{g}, 1 \mu \mathrm{m}$

An approximately fivefold increase of the total number of apoptotic photoreceptors was observed in the 24-weekold RanBP2 ${ }^{+/+}$mice compared with the 12-week-old RanBP2 $2^{+/+}$mice (Figure 4e). In contrast, comparison of 12- and 24-week-old RanBP2 ${ }^{+/-}$mice showed no significant difference in cell death (Figure 4f). Both wild-type and RanBP2 $2^{+/-}$mice had similar total number of apoptotic cells at the age of 12 weeks (Figure 4g), whereas there was a significant increase of apoptosis in wild-type, but not RanBP2 ${ }^{+/-}$mice, at the age of 24 weeks (Figure 4h). On the other hand, similar analyses of the peripheral regions of the retinas showed that there was not a significant agedependent increase to light-elicited degeneration in these regions of the retinas, although such trend was observed (Figure 4i-l). Finally, analysis of cell death in central and peripheral regions of age- and genotype-matched retinas shows that apoptosis is prominent in the central region of the retina (Supplementary Figures 3a-d). Apoptosis increases with age in $\operatorname{RanBP2} 2^{+/+}$, but not RanBP2 $2^{+/-}$mice (Supplementary Figures $3 a-d)$. Collectively, the data show that the central retina presents the highest susceptibility to cell death upon light-elicited stress and haploinsufficiency of RanBP2 blocks this effect.

The expression and SUMOylation of topoisomerase-Il $\alpha$ (Topo II $\alpha$ ) and the levels of opsin apoprotein are not affected by RanBP2 in retinal neurons. RanBP2 was shown physiologically to modulate the SUMOylation of Topo $11 \alpha$ and its localization, ${ }^{32}$ whereas reduced levels of opsin apoprotein of rod photoreceptors protects these neurons from the light-elicited degeneration. ${ }^{9}$ Hence, to gain further insight into the molecular basis of the neuroprotective effects of a deficit of RanBP2 in photoreceptor neurons, we examined whether haploinsufficiency of RanBP2 affected the levels of SUMOylation of Topo Il $\alpha$ and opsin apoprotein in the absence and presence of light stress. Conversely to other tissues and HeLa cells with high mitotic activity, we found that Topo Il $\alpha$ or its sumoylated isoform is not detectable in retinal neurons (Figure 5a) and haploinsufficiency of RanBP2 did not cause a change in the levels of opsin apoprotein 

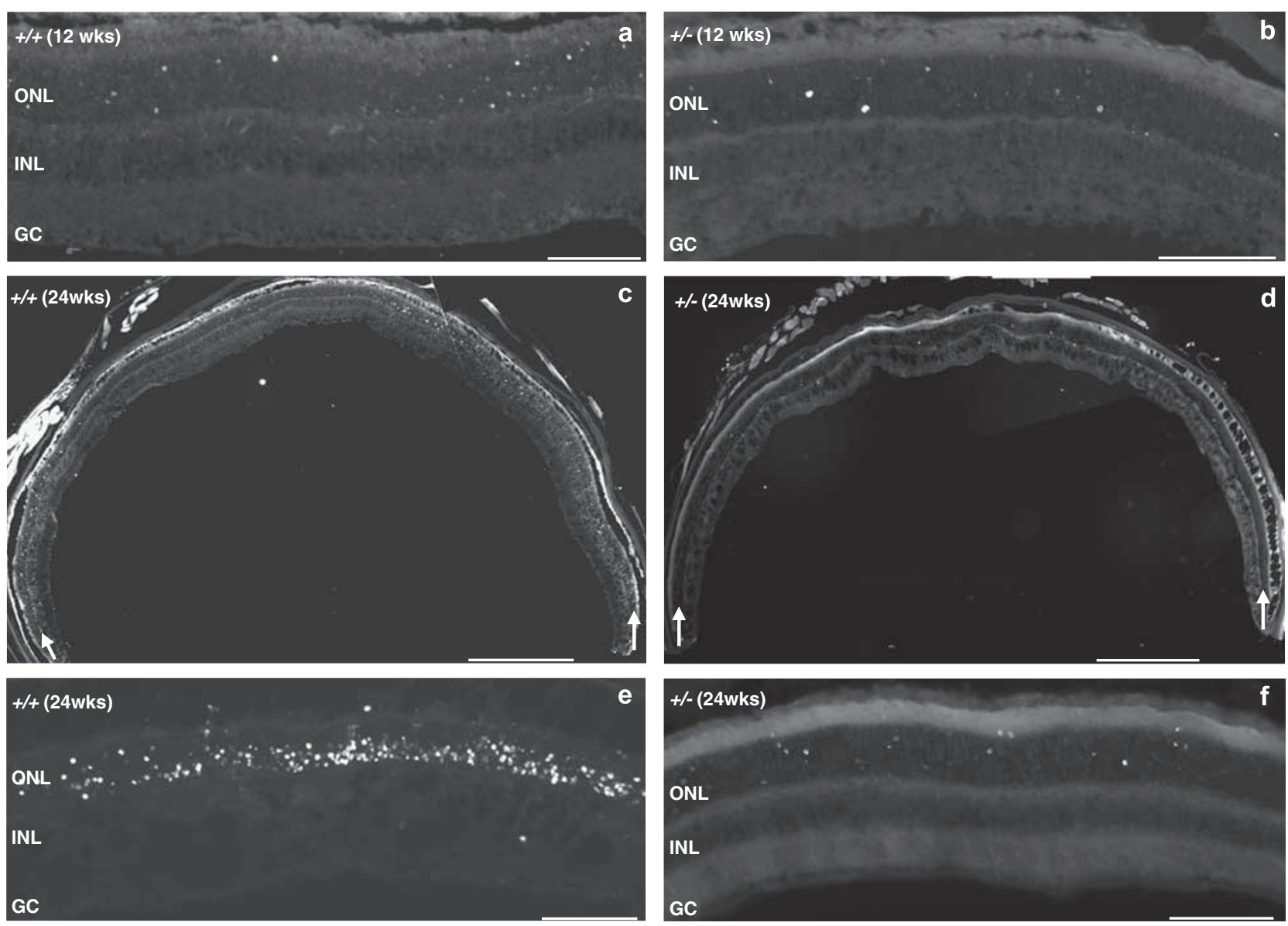

Figure 3 Light-elicited and age-dependent accumulation of apoptotic nuclei selectively in photoreceptors is substantially reduced in RanBP2 haploinsufficient mice. TUNEL staining reflecting the nucleosomal DNA fragmentation of nuclei of photoreceptors shows comparable cell death between 12-week-old RanBP2 $2^{+/+}$and RanBP2 $2^{+/-}$ mice (a and $\mathbf{b}$ ), whereas there is a substantial increase in apoptosis in 24-week-old $\operatorname{RanBP2} 2^{+/+}$, but not $\operatorname{RanBP2} 2^{+/-}$mice $(\mathbf{c}-\mathbf{f})$. $\mathbf{a}, \mathbf{b}, \mathbf{e}$, and $\mathbf{f}$ are high-power micrographs of the central region of the retina. $\mathbf{c}$ and $\mathbf{d}$ are low power micrographs of the whole retina attached to the eyecup (white arrows point to the outer nuclear layer of photoreceptors). $\mathrm{ONL}$, outer nuclear layer (nuclei of photoreceptors); INL, inner nuclear layer (nuclei of second-order neurons); GC, ganglion cell layer. Scale bar in a, b, e, and f, $100 \mu \mathrm{m}$; scale bar in $\mathbf{c}$ and $\mathbf{d}, 500 \mu \mathrm{m}$

under either light condition (Figure 5b). Hence, these data do not support Topo Il $\alpha$ - or opsin-dependent effects promoted by RanBP2 in neuroprotection.

RanBP2 $^{+/-}$mice present a deficit in fat mass. Several lines of evidence from this and earlier works suggest that lipid metabolism may be deregulated in $\operatorname{RanBP}^{+/-}$mice. First, several facets of membrane dysgenesis are significantly alleviated in $\mathrm{RanBP}^{+/-}$compared with RanBP2 ${ }^{+/+}$mice. In particular, the formation of large MLBs and vesicular deposits with single or no discernable membrane leaflet reminiscent of lipid droplets (LDs) structures are observed often in disorders affecting various facets of lipid metabolism or trafficking. ${ }^{33-38}$ Second, RanBP2 haploinsufficient mice present age-dependent decreased weight gain on a high-energy diet. ${ }^{31}$ Finally, gene expression profiling between $\operatorname{RanBP}^{+/+}$and RanBP2 ${ }^{+/-}$mice suggest that lipid metabolism may be deregulated systemically in $\operatorname{RanBP}^{+/-}$mice (unpublished observations). To further probe whether haploinsufficiency of RanBP2 modulated lipid metabolism, RanBP2 ${ }^{+/+}$and RanBP2 ${ }^{+/-}$mice were placed on a high-fat diet (40\%) for
12 weeks. In contrast to $\operatorname{RanBP}^{+/+}$mice, $\operatorname{RanBP}^{+/-}$are remarkably refractory to body weight gain (Figure 6a) and present significantly less accumulation of epididymal fat mass (Figure 6b). These results support that lipogenesis is downregulated in $\mathrm{RanBP}^{+/-}$mice.

Haploinsufficiency of RanBP2, age, and light, promote differential changes in lipid metabolites. Cholesterol and free fatty acids (FFAs), such as docosahexaenoic acid (DHA), are abundant and critical components of membranes of the outer segments of photoreceptors and other neurons, ${ }^{39-43}$ and abnormalities in the level of these have been linked to several syndromic and nonsyndromic retinal dystrophies. ${ }^{34,44-55}$ Moreover, light-elicited lipid peroxidation and deregulation of the production of FFAs (or metabolites thereof) are thought to promote the degeneration of photoreceptors, endothelial cells, and pancreatic $\beta$-cells, by mechanisms that remain largely elusive. ${ }^{10,13,56-60}$ Hence, we examined whether the levels and distribution of cholesterol and FFAs are deregulated in the retina of haploinsufficient RanBP2 mice. As shown in Figure 7, we did not discern changes in cholesterol lipid droplets (LDs) in 
Total cell death of photoreceptors
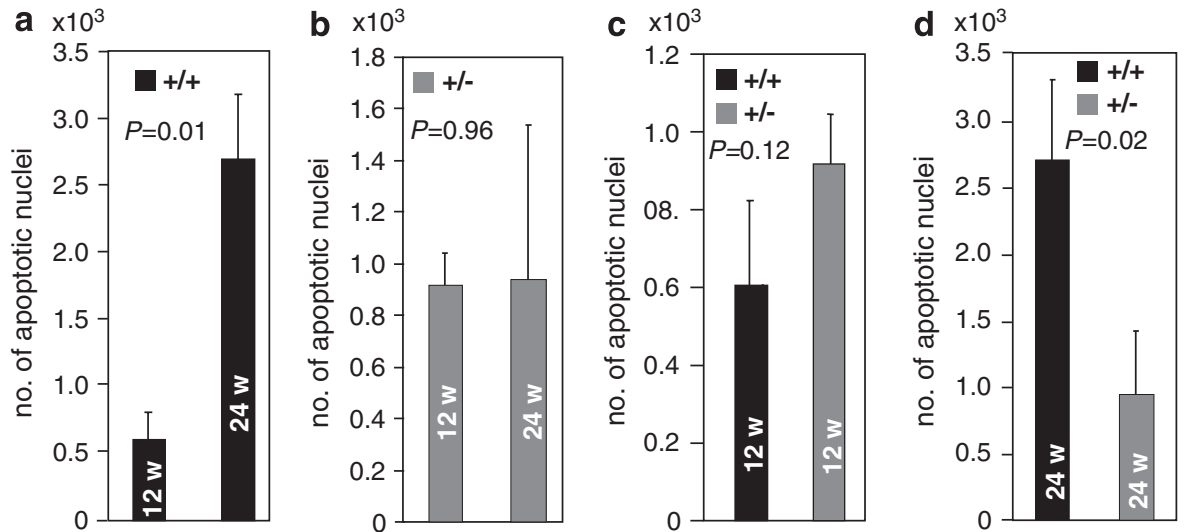

Cell death of photoreceptors in central retina
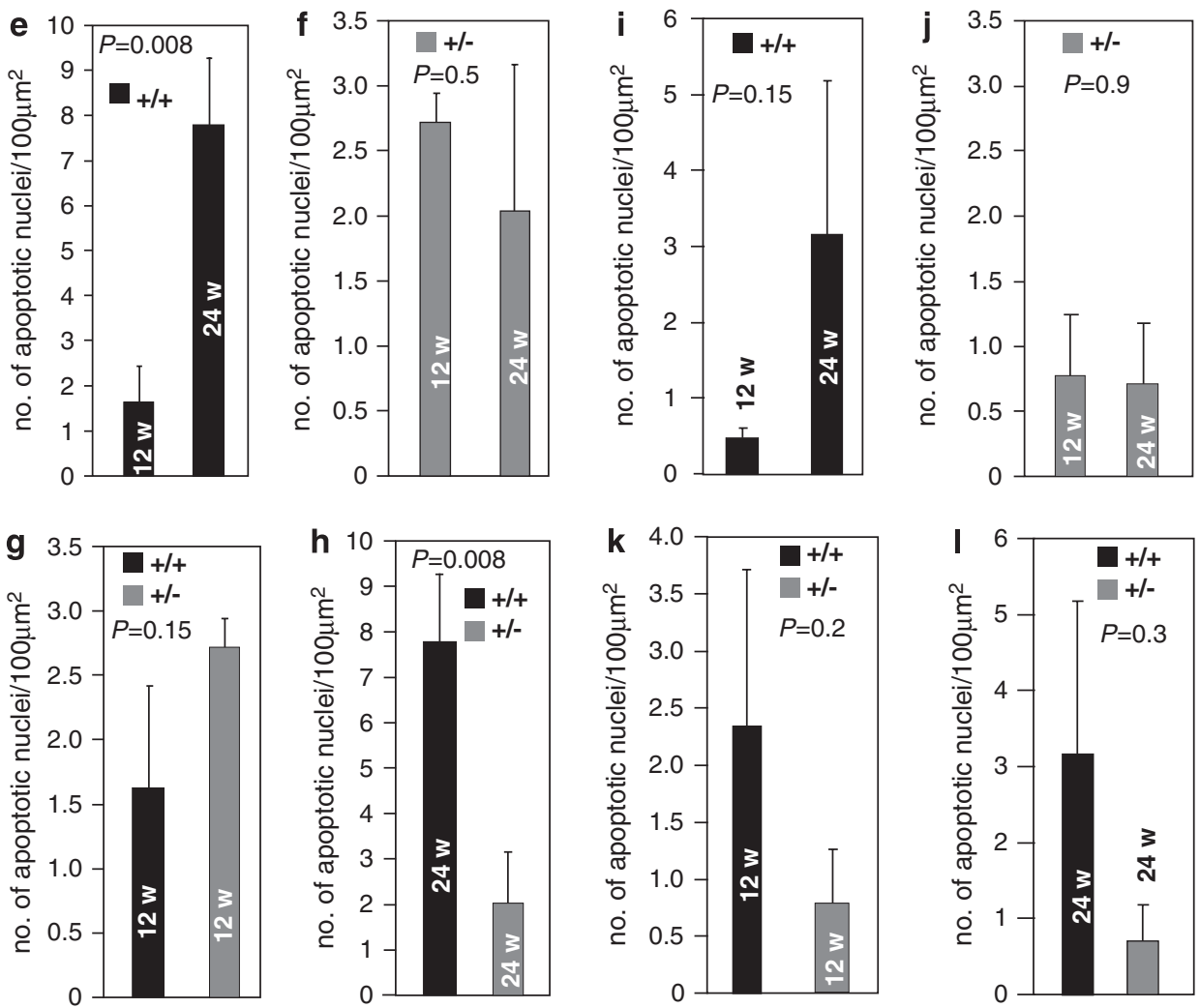

Figure 4 Quantitative morphometric analyses of apoptosis of photoreceptor neurons in 12- and 24-week-old RanBP2 ${ }^{+/+}$and RanBP2 ${ }^{+/-}$mice upon prolonged light exposure. There is an approximately fivefold significant increase in the absolute number of apoptotic nuclei in 24-week-old RanBP2 ${ }^{+/+}$mice compared with 12-week-old ones (a) that is not observed in RanBP2 ${ }^{+/-}$mice (b). At the age of 12 weeks, there was no difference in apoptosis between RanBP2 ${ }^{+/+}$and RanBP2 $2^{+/-}$mice (c), but RanBP2 ${ }^{+/-}$mice had significantly less apoptotic cells than RanBP2 ${ }^{+/+}$mice at the age of 24 weeks (d). There were also significant regional differences in apoptosis between the central (e-h) and peripheral (i-l) sections of retinas. There is an approximately fivefold significant increase in the number of apoptotic nuclei from 12- to 24 -weekold RanBP2 $2^{+/+}$mice (e), but not RanBP2 ${ }^{+/-}$mice (f). No difference in cell death is noticeable between 12 -week-old RanBP2 ${ }^{+/+}$and RanBP2 ${ }^{+/-}$mice $(\mathbf{g})$. At the age of 24 weeks, $R a n B P 2^{+/+}$mice present a significant increase in cell death, which is suppressed in RanBP2 ${ }^{+/}$mice (h). Although not significant, similar trends were observed in the peripheral regions of the retina (i-I). No difference in apoptosis was observed between 12- and 24-week-old RanBP2 ${ }^{+/-}$mice in either the central (f) or the peripheral (j) regions of the retina. Results shown represent the mean \pm S.D. $(n=4)$

photoreceptors between 24-week-old $\operatorname{RanBP2}^{+/+}$and RanBP2 $2^{+/-}$mice in the absence or presence of chronic light stress. However, we noted that the distribution of LDs was disturbed differently in retina pigment epithelium (RPE) cells located adjacent to the tip of the outer segments of
$\operatorname{RanBP2}^{+/+}$and RanBP2 ${ }^{+/-}$mice, but only when these were exposed to light-elicited stress (Figure $7 a-d)$. To this effect, RanBP2 $2^{+/+}$and RanBP2 ${ }^{+/-}$mice on a 12-h light-dark cyclic illumination presented LDs finely distributed throughout the RPE cells (Figure 7a-b). In 


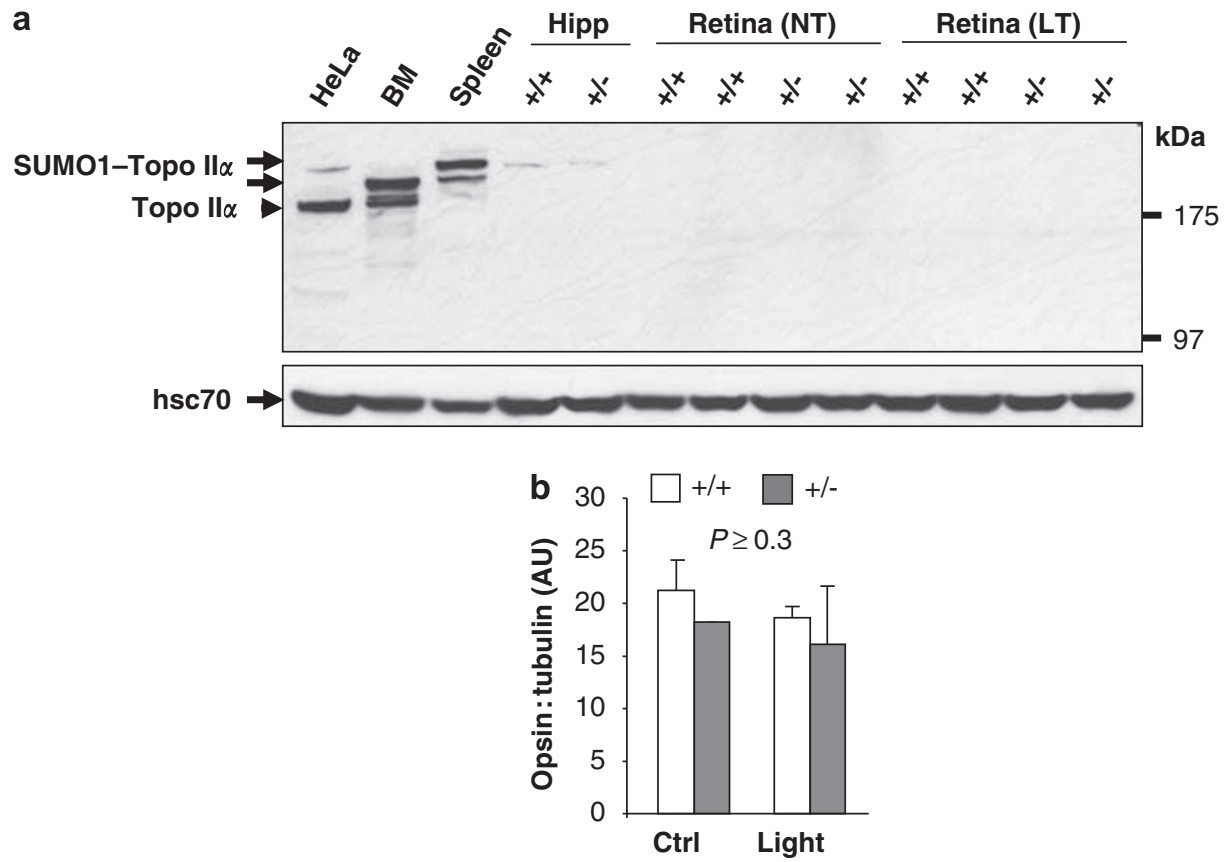

Figure 5 Haploinsufficiency of RanBP2 has no effect on the levels of topoisomerase-II $\alpha$ (Topo Il $\alpha$ ), SUMOylated Topo Il $\alpha$ (SUMO1-Topo Il $\alpha$ ), and apoprotein opsin in the retina. (a) The expression of Topo Il $\alpha$ and sumoylated Topo Il $\alpha$ in retinas of non (NT)- and light-treated (LT) 24-week-old RanBP2 ${ }^{+/+}$and RanBP2 ${ }^{+/-}$mice was assessed by immunoblot analyses of retinal extracts $(100 \mu \mathrm{g})$ and compared with the extracts $(100 \mu \mathrm{g})$ of HeLa, bone marrow (BM) from the femoral bone, spleen, and hippocampus (Hipp). Regardless of the light-treatment and genotype, Topo Il $\alpha$ and sumoylated Topo ll $\alpha$ were not detected in the retinas, but they were expressed in HeLa, $\mathrm{BM}$, and spleen, whereas traces of sumoylated Topo Il $\alpha$ were detected in the hippocampus. Blot was reprobed for the cytosolic heat-shock 70 protein (hsc70) as loading control. (b) The expression of the apoprotein opsin in retinas of 24-week-old $R_{a n B P 2}{ }^{+/+}$and RanBP2 ${ }^{+/}$mice under normal cyclic (Ctrl) and prolonged light exposure (light) was quantified from immunoblot analyses of retinal extracts $(2 \mu \mathrm{g})$ by densitometry and normalized for tubulin expression. No changes in apoprotein opsin expression were observed regardless of the exposure to light and genotype. Results shown represent the mean \pm S.D. $(n=4)$. AU, arbitrary units
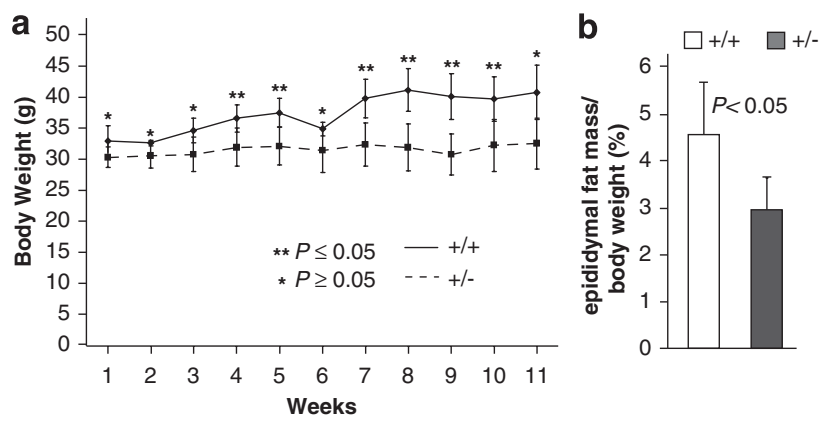

Figure 6 Haploinsufficiency of RanBP2 renders 24-week-old mice refractory to the gain of body weight and epididymal fat mass when placed on a high-fat $(40 \%)$ diet. RanBP2 ${ }^{+/+}$, but not RanBP2 ${ }^{+/-}$mice, gain significant body weight (a) and epididymal fat mass (b) when placed on a high-fat diet for 12 weeks. In (a) double asterisks represent significant differences between the groups (Student's $t$-test, $P \leqslant 0.05$ ). Statistical significance was also found across the time course of the experiment (repeated measures of two-way ANOVA, $P=0.03$ ). Results shown represent the mean \pm S.D. $(n=4)$, ANOVA, analysis of variance

contrast, upon light-elicited stress, the LD deposits (aggregates) were seen uniformly distributed in the RPE of RanBP2 $2^{+/+}$mice (Figure 7c), whereas such deposits were localized prominently to the basal end of RPE cells of RanBP2 $^{+/-}$mice (Figure 7d). Examination of cholesterol and FFAs content of retinas shows that neither the level of total nor free cholesterol was changed between 24-week-old $\mathrm{RanBP}^{+/+}$and $\mathrm{RanBP}^{+/-}$mice regardless of the light exposure, although there was a trend for $\operatorname{RanBP} 2^{+/-}$mice to present lower levels of total and free (nonesterified) cholesterol on a normal 12-h light-dark cyclic illumination (Figure $7 e$ and $\mathrm{f}$ ). In contrast, we found that the content of FFA in the retina varied significantly with age and genotype of RanBP2 mice in a light-independent fashion. There was no difference in FFA content between 12-week-old RanBP2 $2^{+/+}$ and $\operatorname{RanBP2}^{+/-}$mice (Figure $7 \mathrm{~g}$ ), whereas 24-week-old RanBP2 $2^{+/}$mice presented reduced levels ( $35-40 \%$ ) of FFA compared with RanBP2 ${ }^{+/+}$(Figure 7h). Finally, we probed further whether prolonged exposure to light promotes oxidative stress in the retina in a light- and genotypedependent manner, as such stress insult is thought to play a role in the degeneration of photoreceptors. ${ }^{10,13}$ As shown in Figure $7 \mathrm{i}$, there was a significant increase of hydrogen peroxide $\left(\mathrm{H}_{2} \mathrm{O}_{2}\right)$ production in retinal neurons upon lightelicited stress and the levels of $\mathrm{H}_{2} \mathrm{O}_{2}$ production did not vary with the genotype of RanBP2 mice.

\section{Discussion}

Our studies reveal an age-dependent switch between 12- and 24-week-old mice on the 129P2/OlaHsd background that significantly increases the susceptibility of photoreceptors to light-induced degeneration and membrane dysgenesis of photoreceptor neurons. RanBP2 haploinsufficiency significantly blocks this switch thus rendering older photoreceptors much less susceptible to light-elicited degeneration and suppressing several facets of membrane dysgenesis, such as the formation of MLBs and the disruption of nascent disk 

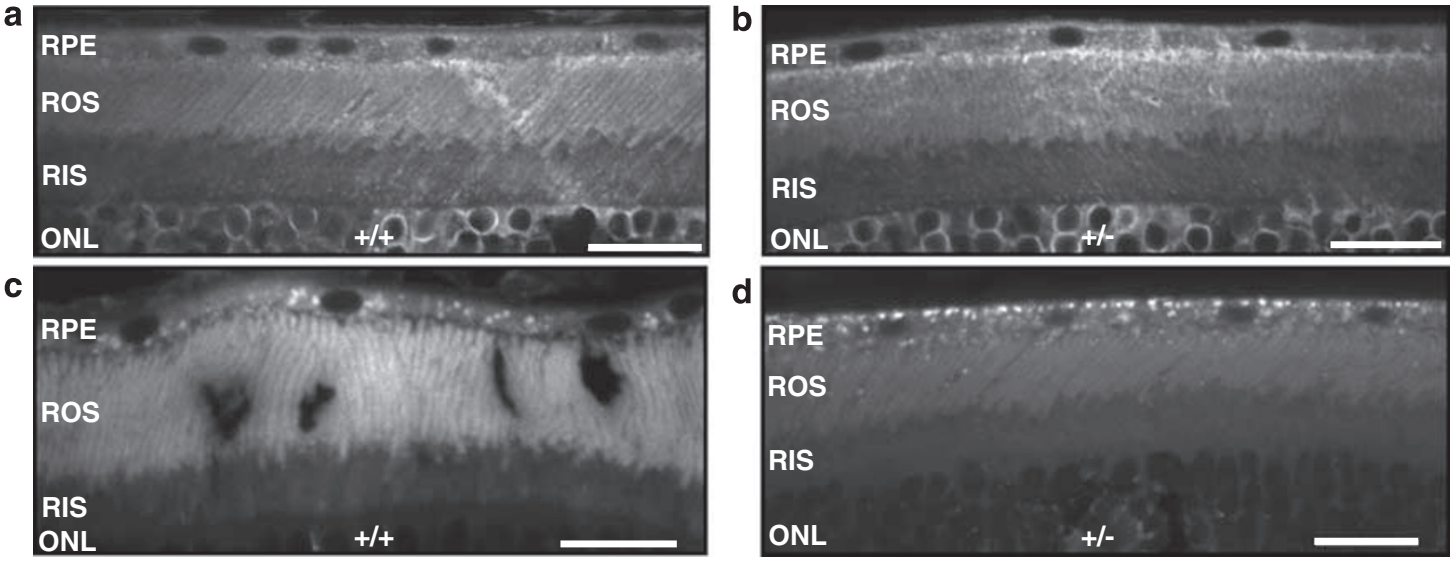

d

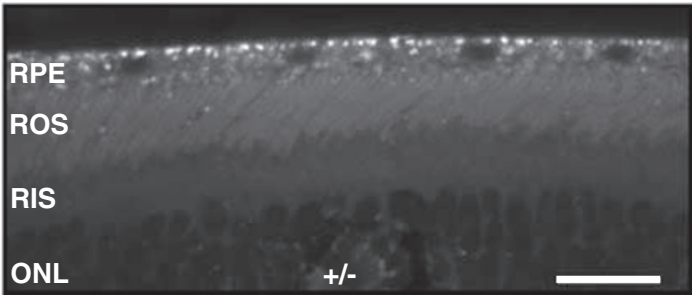

e

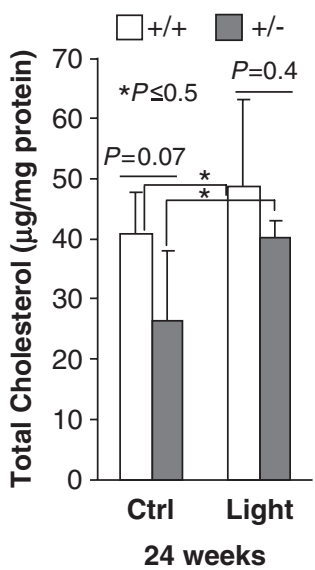

f

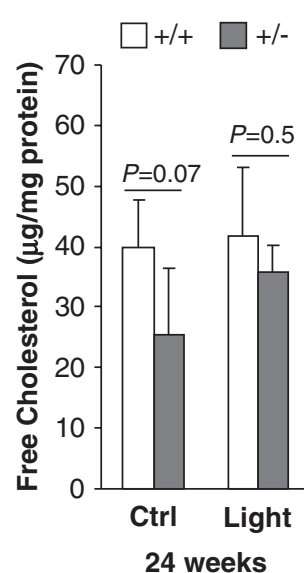

g

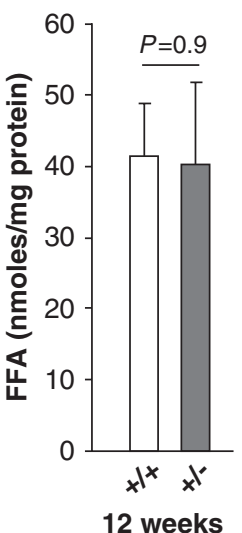

h

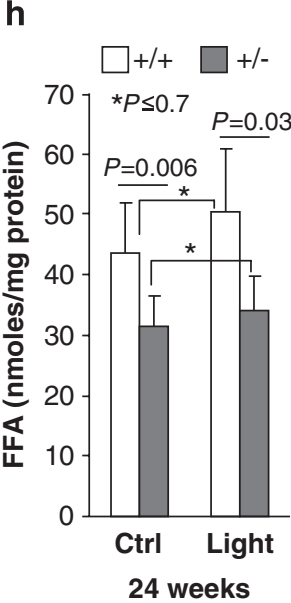

i $\square+/+\square+/-$

$$
\begin{array}{rr}
\times 10^{-2} \quad * P \leq 0.01 \\
0
\end{array} \quad * * P \leq 0.02
$$

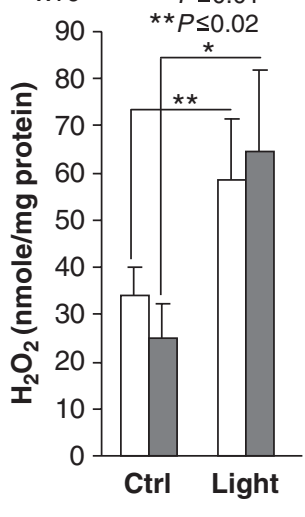

Figure 7 Lipid metabolic deficits and reactive oxygen species production in 24-week-old RanBP2 ${ }^{+/+}$and RanBP2 ${ }^{+/-}$mice upon light-elicited stress. Filipin staining of cholesterol/lipid droplets (LDs) shows that $\operatorname{RanBP}^{+/+}$(a) and $\operatorname{RanBP}^{+/-}$(b) under normal cyclic light present no visible difference in LD, whereas under light-elicited stress, there is an apparent coalescence of LD throughout the perikarya of the retinal pigment epithelium (RPE) in RanBP2 ${ }^{+/+}$mice (c) and coalescence and polarized localization of LD to the basal end of the RPE of RanBP2 ${ }^{+/-}$mice (d). No significant and discernable changes in LD distribution were observed in the outer (ROS) and inner segments (RIS) of rod photoreceptor neurons (a-d). Total (e) and free (f) cholesterol were not significantly altered in retinas of RanBP2 $2^{+/+}$and RanBP2 $2^{+/-}$mice regardless of the light treatment, although RanBP2 ${ }^{+/-}$mice exhibited a trend to present lower levels of total $(\mathbf{e})$ and free $(\mathbf{f})$ cholesterol than RanBP2 ${ }^{+/+}$mice when reared under cyclic light, but not prolonged light exposure $(n=4)$. The content of free fatty acids $(F F A)$ in the retina remained unaltered between RanBP2 $2^{+/+}$and $R a n B P 2^{+/-}$mice at the age of 12-weeks $(n=4)(\mathbf{g})$, whereas 24-week-old RanBP2 ${ }^{+/-}$mice presented approximately $35 \%$ decreased levels of FFA than RanBP2 ${ }^{+/+}$mice and this remained unchanged under prolonged light exposure ( $n=7$ for RanBP2 ${ }^{+/+}$mice under normal cyclic light, $n=6$ for RanBP2 ${ }^{+/}$mice under normal cyclic light, $n=4$ for mice under prolonged light exposure; $\mathbf{h}$ ). (i) The level of hydrogen peroxide $\left(\mathrm{H}_{2} \mathrm{O}_{2}\right)$ increases in retinas upon light-elicited stress regardless of the genotype of RanBP2 mice $(n=4)$. Results shown represent the mean \pm S.D. ROS, rod outer segment (compartment) of photoreceptors; RIS, rod inner segment (compartment) of photoreceptors; ONL, outer nuclear layer (nuclei of photoreceptors); RPE, retinal pigment epithelium; Ctrl, control mice placed under light-dark cyclic illumination ( $<70$ lux); Light, mice placed under prolonged light exposure (48 h; 1200 lux)

formation. Strikingly, a genotype-dependent, but light-independent, decrease in FFA of the retina in 24-week-old, but not 12 -week-old, RanBP2 ${ }^{+/-}$mice correlates well with the morphological phenotypes observed upon light-elicited stress. Hence, the data support a model whereby a RanBP2-dependent reduction of FFA content renders neuroprotection to photoreceptor neurons to light-induced damage (Figure 8). The decrease of FFAs in the retina of RanBP2 $2^{+/-}$ mice, and the resistance to weight gain and formation of adipose tissue of $\mathrm{RanBP}^{+/-}$mice when placed on a high-fat diet suggest that FFA uptake is compromised in RanBP2 $2^{+/-}$ mice. The deregulation of FFA uptake may also affect the transport (and distribution) of cholesterol in $\mathrm{LDs}^{61}$ as observed in retina pigment epithelial cells of RanBP2 $^{+/-}$ mice, a phenotype likely secondary to deficits in FFA. Lower levels of FFA in FFA-enriched photoreceptor neurons likely decreases lipid peroxidation, such as formation of fatty acid hydroperoxides, which are implicated in the propagation of lipid peroxidation. $^{62}$ The major outcomes of lipid peroxidation in photoreceptors are twofold. First, it promotes the generation of toxic FFA metabolites, which by themselves or in concert with other ligands, promote apoptosis. ${ }^{60,63-65}$ Second, changes in the orientation of the acyl chain of peroxidized fatty acids toward the hydrophilic exterior of the lipid bilayer (e.g., lipid whisker model $^{66}$ ) may disrupt critical protein-protein and protein-lipid contacts required for the membrane biogenesis of nascent disks of photoreceptors or even generate novel pathological ligand(s) contributing to the death of photoreceptors.

The light-elicited pathways and mechanisms leading to the apoptosis of photoreceptors remain largely elusive. 


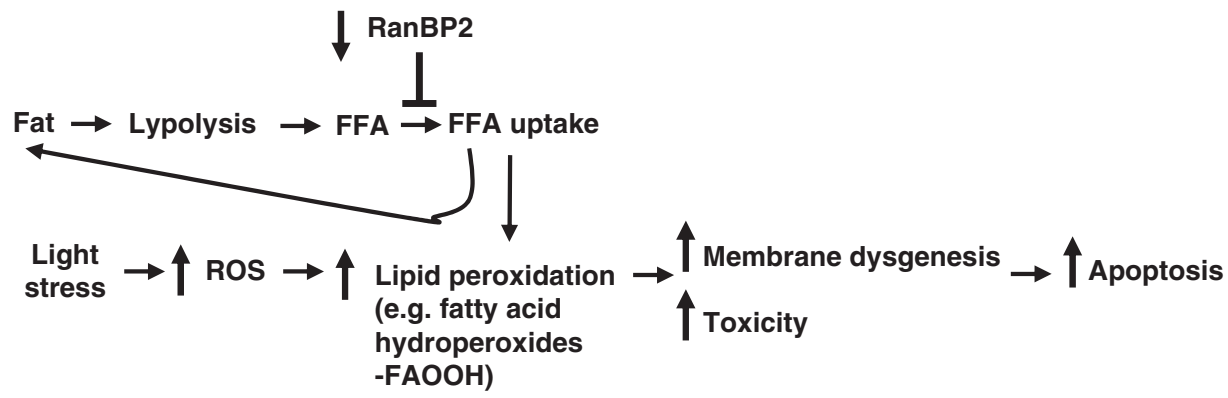

Figure 8 Model of the effect of haploinsufficiency of RanBP2 and light-elicited stress in the production of free fatty acids (FFAs), lipid peroxidation by reactive oxygen species (ROS), and downstream effect of these in membrane dysgenesis, toxicity, and stimulation of apoptosis (see text for details). Upward arrows indicate an increased level/effect; downward arrow indicates a decreased level/effect

In particular, the identity of the FFA or metabolites thereof underlying neuroprotection and exacerbating apoptosis in the central region of the retina upon light-induced damage are unknown. In light of the large content of DHA in the membrane of the outer segment of photoreceptors, ${ }^{43}$ $\mathrm{DHA}$ is a strong candidate to mediate neuroprotection when in deficit or to stimulate apoptosis when at high levels. Indeed, a diet deficit in the linolenic acid precursor of DHA of albino rats confers neuroprotection to photoreceptor neurons upon light-induced damage. ${ }^{67}$ Collectively, these observations support that photoreceptors contain an excessive pool of DHA with deleterious implications upon light-elicited damage and that the bioavailability of FFA to photoreceptors differs between the central and peripheral regions of the retina. On the other hand, light-elicited degeneration of photoreceptors of Drosophila because of deficits in phosphatidylinositolphospholipase C- $\beta 4$ (PI-PLC $\beta 4$ )-mediated production of diacylglycerol (DAG) ${ }^{68}$ and generation of FFA and metabolites thereof from DAG by a DAG lipase encoded by inaE, ${ }^{59}$ suggest another vital role of FFA signaling in the survival of photoreceptor neurons (and Drosophila). Although it remains unclear whether such PI-PLC-dependent signaling pathway is conserved in vertebrate photoreceptor neurons, the mammalian homolog of the PI-PLC- $\beta 4$ of Drosophila is present in photoreceptor neurons. $^{69,70}$ Hence, this PI-PLC signaling pathway may be another source for the generation of FFA from the breakdown of phosphatidylinositol 4,5-biphosphate $\left(\mathrm{PIP}_{2}\right)^{71-79}$

Finally, analysis of haploinsufficiency of RanBP2 greatly facilitates the identification and dissection of primary phenotypes from confounding secondary phenotypes, which are often difficult to parse in severe phenotypes. ${ }^{31,32}$ However, genetic tools to manipulate and analyze a diverse but limited set of partners, each associating specifically to selective domains of RanBP2, will provide novel insights into what specific biological activities directly linked to each of the functions of RanBP2 $2^{20-26,31}$ confers light-elicited neuroprotection and modulates lipid metabolism. The data here presented do not suggest a direct role of sumoylation of Topo $11 \alpha^{32}$ and opsin apoprotein ${ }^{9}$ in such processes because of the absence of the former from retinal neurons and the lack of change in the levels of the latter in $\operatorname{RanBP}^{+/}$mice, respectively. It is of interest to note that an autosomal dominant acute necrotizing encephalopathy triggered by the onset of a febrile illness in the human maps to $2 q 12.1-2 q 13$, where RanBP2 is localized. ${ }^{80-82}$ Identification of such potential genetic lesion(s) in RanBP2 may provide new clues of the role of RanBP2 and its partners in neurological function and cell death upon various stressors. Regardless, the RanBP2 mouse model defines a new genetic tool and framework to partition, isolate, and define, molecularly and genetically, activities and subcellular processes linked to RanBP2 and aging that are important in modulating the survival of photoreceptors and other neurons, and manifestations of aging-related diseases affecting the retina (e.g. macular dystrophies) and elsewhere, upon exposure to deleterious stressors.

\section{Materials and Methods}

Mice. RanBP2 $2^{+/-}$mice were described elsewhere. ${ }^{31}$ RanBP2 $^{+/+}$and RanBP2 ${ }^{+/}$mice were in an inbred 129P2/OlaHsd background and reared in 70 lux of diffused white fluorescent light and 12:12 light-dark cycle. Mice were fed with a standard chow diet ( 10\% fat; test diet 5LJ5, Purina) or placed whenever applicable on a high-fat ( $\sim 40 \%)$ diet (test diet DIO 58Y1, Purina) for 12 weeks. Animal protocols were approved by the Institutional Animal Care and Use Committee at Duke University and the procedures adhered to the ARVO guidelines for the Use of Animals in Vision Research.

Prolonged light exposure treatment. Mice were placed in a white reflective cage with food and water and fluorescent light bulbs mounted at the top of the cage. Illuminance was measured at the bottom of the cage with a traceable dualrange light meter (Fisher Scientific). Mice were exposed to 1200 lux of continuous, cooled, and diffused fluorescent white light for $48 \mathrm{~h}$. Mice were killed and the eyeballs immediately collected and processed for histology and morphometric analyses.

Histology. Eyes were prepared for light and electron microscopy by fixing eyecups overnight in $2 \%$ glutaraldehyde and $2 \%$ paraformaldehyde in $0.1 \%$ cacodylate buffer, $\mathrm{pH} 7.2$ at $4{ }^{\circ} \mathrm{C}$. Semithin sections $(0.5 \mu \mathrm{m})$ along the vertical meridian were mounted on slides and stained with $1 \%$ methylene blue. Light images of the retina were acquired with a Nikon C1-Plus light microscope equipped with Nomarski optics and coupled to a SPOT RT-SE digital camera (Diagnostic Instruments)

TUNEL staining and quantitative morphometric analysis of apoptosis. Apoptosis was detected in situ with the DeadEnd Fluorometric TUNEL System (Promega) on retinas fixed with $4 \%$ paraformaldehyde. Images of TUNEL-positive nuclei of sections of eyecups were captured with a laser confocal Nikon C1-Plus light microscope equipped with epifluorescence and coupled to a Cascade $1 \mathrm{~K}$ digital camera (Roper Scientific). Images were acquired with $\times 4$ and $\times 10$ objectives. Digital stitching of composite images acquired with the $\times 4$ objective was performed with Photoshop CS (Adobe). Quantitative morphometric analyses of images analyzed with Metamorph v6.2 (Molecular Devices) were performed on eight sections per eye along the vertical meridian with $a \times 10$ objective. Typically, images of the whole section of the retina comprised 7-8 image 
fields. Peripheral images of the retina containing the marginal areas of the retina comprised the first and last image fields. Central regions of the retina comprised typically the third and fourth image fields of the retina. Statistical analysis of apoptosis was performed with two-tailed equal variance $t$-test.

Transmission electron microscopy. Posterior eyes were processed as described in the Histology section followed by postfixation in $2 \%$ osmium tetraoxide in $0.1 \%$ cacodylate buffer and embedded in Spurr resin. Sixty-five nanometer thick sections were cut with an ultramicrotome and stained with uranyl acetate and lead citrate. Specimens were visualized with JEOL 1200 EX and Philips CM 12 transmission electron microscopes. Low magnification images were captured with the Philips TEM coupled with an AMT camera and processed with the Image Capturing Engine software (version 5.42355). High magnification images were captured with the JEOL TEM and negatives were scanned with Photoshop CS.

Immunoblot analyses. Retina samples were homogenized with a Kontes Microtube Pellet Pestle Rods with Motor in NP-40 buffer $(50 \mathrm{mM}$ Tris- $\mathrm{HCl}, \mathrm{pH} 8$ $150 \mathrm{mM} \mathrm{NaCl}, 1 \%$ Nonidet P-40 (NP-40), with complete protein inhibitor cocktail (Roche Applied Science, Indianapolis, IN, USA). Samples were centrifuged at $10000 \mathrm{~g}$ for $15 \mathrm{~min}$ and supernatants collected. Protein concentration was measured by Bradford method using BSA as standard. Protein extracts were resolved by SDS-polyacrylamide gel electrophoresis (SDS-PAGE) and Western blotting was carried out as described elsewhere. ${ }^{83}$ Primary antibodies used: Mouse anti-acetylated tubulin (Sigma, 1:40000), rabbit anti-rhodopsin (Affinity Bioreagents, $1: 20000$ ), rabbit anti-Hsc70 (Stressgen, $1: 3000$ ), rabbit anti-Topo $\| \alpha$ (Topogen, $1: 3000$ )

Filipin staining. Radial retinal cryosections ( $\sim 10 \mu \mathrm{m}$ thick) mounted on slides were rinsed three times with phosphate-buffered saline (PBS) pH 7.4 and incubated with $0.05 \mathrm{mg} / \mathrm{ml}$ of filipin (Sigma) for $2 \mathrm{~h}$ at room temperature in the dark. Specimens were then rinsed three times with PBS and mounted with ProLong Gold antifade (Invitrogen)

Hydrogen peroxide measurement. The hydrogen peroxide levels were assayed using the Amplex red hydrogen peroxide/peroxidase assay kit (Invitrogen) as per the manufacturer's instructions. In all, $10 \mu \mathrm{l}$ of NP40 extract was used for each measurement. Results were normalized against soluble protein contents in the extract. Two-tailed equal variance $t$-test statistical analysis was performed.

Free cholesterol and total cholesterol quantitation. Free cholesterol and total cholesterol were determined with the cholesterol and cholesterol ester quantitation kit (Biovision, Mountain View, CA, USA) as per the manufacture's instructions. Briefly, the $3 \mu$ l retinal NP40 extracts were diluted with assay buffer for measurement by fluorescence (excitation/emission/cutoff $=560 / 590 / 590 \mathrm{~nm}$; SpectraMax M5, Molecular Devices). Total cholesterol was measured with cholesterol esterase added; free cholesterol was measured without cholesterol esterase. Results were normalized to soluble protein content in the extracts. Twotailed equal variance $t$-test statistical analysis was performed.

FFAs quantification. FFAs were determined with the FFA Quantification kit (Biovision, Mountain View, CA, USA) to detect C-8 (octanoate) and longer fatty acids as per the manufacture's instructions. Briefly, retinal NP40 extracts were used for each reaction. FFA content was measured by a colorimetric assay (Ab570) with palmitic acid employed as a standard. FFA results were normalized against soluble protein contents in the extract. Two-tailed equal and unequal variance $t$-test statistical analysis was performed.

Acknowledgements. This work was supported by NIH Grant EY11993 and Pearle Vision Foundation to PAF and NIH 2P30-EY005722-21. AY was supported by undergraduate research scholarships from Howard Hughes Medical Institute and American Federation Aging Research. PAF is the Jules \& Doris Stein Research to Prevent Blindness Professor.

1. Pacione LR, Szego MJ, Ikeda S, Nishina PM, McInnes RR. Progress toward understanding the genetic and biochemical mechanisms of inherited photoreceptor degenerations. Annu Rev Neurosci 2003; 26: 657-700.
2. Reme CE. The dark side of light: rhodopsin and the silent death of vision the proctor lecture. Invest Ophthalmol Vis Sci 2005; 46: 2671-2682.

3. Danciger M, Lyon J, Worrill D, LaVail MM, Yang H. A strong and highly significant QTL on chromosome 6 that protects the mouse from age-related retinal degeneration. Invest Ophthalmol Vis Sci 2003; 44: 2442-2449.

4. Danciger M, Yang H, Ralston R, Liu Y, Matthes MT, Peirce J et al. Quantitative genetics of age-related retinal degeneration: a second F1 intercross between the $\mathrm{A} / \mathrm{J}$ and $\mathrm{C} 57 \mathrm{BL} / 6$ strains. Mol Vis 2007; 13: 79-85.

5. Hao W, Wenzel A, Obin MS, Chen CK, Brill E, Krasnoperova NV et al. Evidence for two apoptotic pathways in light-induced retinal degeneration. Nat Genet 2002; 32: 254-260.

6. Grimm C, Wenzel A, Williams T, Rol P, Hafezi F, Reme C. Rhodopsin-mediated blue-light damage to the rat retina: effect of photoreversal of bleaching. Invest Ophthalmol Vis Sci 2001; 42: 497-505.

7. Wenzel A, Reme CE, Williams TP, Hafezi F, Grimm C. The Rpe65 Leu450Met variation increases retinal resistance against light-induced degeneration by slowing rhodopsin regeneration. J Neurosci 2001; 21: 53-58.

8. Woodruff ML, Wang Z, Chung HY, Redmond TM, Fain GL, Lem J. Spontaneous activity of opsin apoprotein is a cause of Leber congenital amaurosis. Nat Genet 2003; 35: 158-164.

9. Grimm C, Wenzel A, Hafezi F, Yu S, Redmond TM, Reme CE. Protection of Rpe65deficient mice identifies rhodopsin as a mediator of light-induced retinal degeneration. Nat Genet 2000; 25: 63-66.

10. Hollyfield JG, Bonilha VL, Rayborn ME, Yang X, Shadrach KG, Lu L et al. Oxidative damage-induced inflammation initiates age-related macular degeneration. Nat Med 2008; 14: $194-198$

11. Linsenmeier RA. Effects of light and darkness on oxygen distribution and consumption in the cat retina. J Gen Physiol 1986; 88: 521-542.

12. Wright AF, Jacobson SG, Cideciyan AV, Roman AJ, Shu X, Vlachantoni $D$ et al. Lifespan and mitochondrial control of neurodegeneration. Nat Genet 2004; 36: 1153-1158.

13. Imamura Y, Noda S, Hashizume K, Shinoda K, Yamaguchi M, Uchiyama S et al. Drusen, choroidal neovascularization, and retinal pigment epithelium dysfunction in SOD1-deficient mice: a model of age-related macular degeneration. Proc Natl Acad Sci USA 2006; 103 11282-11287.

14. Grimm C, Wenzel A, Stanescu D, Samardzija M, Hotop S, Groszer M et al. Constitutive overexpression of human erythropoietin protects the mouse retina against induced but not inherited retinal degeneration. J Neurosci 2004; 24: 5651-5658.

15. Cideciyan AV, Hood DC, Huang Y, Banin E, Li ZY, Stone EM et al. Disease sequence from mutant rhodopsin allele to rod and cone photoreceptor degeneration in man. Proc Nat Acad Sci USA 1998; 95: 7103-7108.

16. Galy A, Roux MJ, Sahel JA, Leveillard T, Giangrande A. Rhodopsin maturation defects induce photoreceptor death by apoptosis: a fly model for RhodopsinPro23His human retinitis pigmentosa. Hum Mol Genet 2005; 14: 2547-2557.

17. Naash ML, Peachey NS, Li ZY, Gryczan CC, Goto Y, Blanks J et al. Light-induced acceleration of photoreceptor degeneration in transgenic mice expressing mutant rhodopsin. Invest Ophthalmol Vis Sci 1996; 37: 775-782.

18. Organisciak DT, Darrow RM, Barsalou L, Kutty RK, Wiggert B. Susceptibility to retinal light damage in transgenic rats with rhodopsin mutations. Invest Ophthalmol Vis Sci 2003; 44 486-492.

19. Vaughan DK, Coulibaly SF, Darrow RM, Organisciak DT. A morphometric study of lightinduced damage in transgenic rat models of retinitis pigmentosa. Invest Ophthalmol Vis $\mathrm{Sci}$ 2003; 44: 848-855.

20. Yokoyama N, Hayashi N, Seki T, Pante N, Ohba T, Nishii K et al. A giant nucleopore protein that binds Ran/TC4. Nature 1995; 376: 184-188.

21. Bernad R, van der Velde H, Fornerod M, Pickersgill H. Nup358/RanBP2 attaches to the nuclear pore complex via association with Nup88 and Nup214/CAN and plays a supporting role in CRM1-mediated nuclear protein export. Mol Cell Biol 2004; 24: 2373-2384.

22. Singh BB, Patel HH, Roepman R, Schick D, Ferreira PA. The zinc finger cluster domain of RanBP2 is a specific docking site for the nuclear export factor, exportin-1. J Biol Chem 1999; 274: 37370-37380

23. Cho KI, Cai Y, Yi H, Yeh A, Aslanukov A, Ferreira PA. Association of the Kinesin-Binding Domain of RanBP2 to KIF5B and KIF5C Determines Mitochondria Localization and Function. Traffic 2007; 8: 1722-1735.

24. Yi H, Friedman J, Ferreira PA. The cyclophilin-like domain of Ran-binding protein-2 modulates selectively the activity of the ubiquitin-proteasome system and protein biogenesis. J Biol Chem 2007; 282: 34770-34778.

25. Ferreira PA, Hom JT, Pak WL. Retina-specifically expressed novel subtypes of bovine cyclophilin. J Biol Chem 1995; 270: 23179-23188

26. Ferreira PA, Nakayama TA, Pak WL, Travis GH. Cyclophilin-related protein RanBP2 acts as chaperone for red/green opsin. Nature 1996; 383: 637-640.

27. Lee GW, Melchior F, Matunis MJ, Mahajan R, Tian Q, Anderson P. Modification of Ran GTPase-activating protein by the small ubiquitin-related modifier SUMOrequires Ubc9, an E2-type ubiquitin-conjugating enzyme homologue. J Biol Chem 1998; 273: $6503-6507$.

28. Mahajan R, Delphin C, Guan T, Gerace L, Melchior F. A small ubiquitin-related polypeptide involved in targeting RanGAP1 to nuclear pore complex protein RanBP2. Cell 1997; 88: 97-107.

29. Mahajan R, Gerace L, Melchior F. Molecular characterization of the SUMO-1 modification of RanGAP1 and its role in nuclear envelope association. J Cell Biol 1998; 140: 259-270. 
30. Matunis MJ, Coutavas E, Blobel G. A novel ubiquitin-like modification modulates the partitioning of the Ran-GTPase-activating protein RanGAP1 between the cytosol and the nuclear pore complex. J Cell Biol 1996; 135: 1457-1470.

31. Aslanukov A, Bhowmick R, Guruju M, Oswald J, Raz D, Bush RA et al. RanBP2 Modulates Cox11 and Hexokinase I Activities and Haploinsufficiency of RanBP2 Causes Deficits in Glucose Metabolism. PLoS Genet 2006; 2: e177.

32. Dawlaty MM, Malureanu L, Jeganathan KB, Kao E, Sustmann C, Tahk S et al. Resolution of sister centromeres requires RanBP2-mediated SUMOylation of topoisomerase Ilalpha. Cell 2008; 133: 103-115.

33. German DC, Liang CL, Song T, Yazdani U, Xie C, Dietschy JM. Neurodegeneration in the Niemann-Pick C mouse: glial involvement. Neuroscience 2002; 109: 437-450.

34. Karan G, Lillo C, Yang Z, Cameron DJ, Locke KG, Zhao Y et al. Lipofuscin accumulation, abnormal electrophysiology, and photoreceptor degeneration in mutant ELOVL4 transgenic mice: a model for macular degeneration. Proc Natl Acad Sci USA 2005; 102: 4164-4169.

35. Lakkaraju A, Finnemann SC, Rodriguez-Boulan E. The lipofuscin fluorophore A2E perturbs cholesterol metabolism in retinal pigment epithelial cells. Proc Natl Acad Sci USA 2007; 104: 11026-11031.

36. Patel SC, Suresh S, Kumar U, Hu CY, Cooney A, Blanchette-Mackie EJ et al. Localization of Niemann-Pick $\mathrm{C} 1$ protein in astrocytes: implications for neuronal degeneration in Niemann- Pick type C disease. Proc Natl Acad Sci USA 1999; 96: 1657-1662.

37. Phillips SE, Woodruff III EA, Liang P, Patten M, Broadie K. Neuronal loss of Drosophila $\mathrm{NPC1a}$ causes cholesterol aggregation and age-progressive neurodegeneration. $J$ Neurosci 2008; 28: 6569-6582.

38. Schmitz G, Muller G. Structure and function of lamellar bodies, lipid-protein complexes involved in storage and secretion of cellular lipids. J Lipid Res 1991; 32 1539-1570.

39. Anderson RE. Lipids of ocular tissues. IV. A comparison of the phospholipids from the retina of six mammalian species. Exp Eye Res 1970; 10: 339-344.

40. Andrews LD, Cohen Al. Freeze-fracture evidence for the presence of cholesterol in particle-free patches of basal disks and the plasma membrane of retinal rod outer segments of mice and frogs. J Cell Biol 1979; 81: 215-228.

41. O'Brien JS, Sampson EL. Fatty acid and fatty aldehyde composition of the major brain lipids in normal human gray matter, white matter, and myelin. J Lipid Res 1965; 6 : 545-551.

42. Albert AD, Boesze-Battaglia $K$. The role of cholesterol in rod outer segment membranes. Prog Lipid Res 2005; 44: 99-124.

43. Fliesler SJ, Anderson RE. Chemistry and metabolism of lipids in the vertebrate retina. Prog Lipid Res 1983; 22: 79-131.

44. Anderson RE, Maude MB, Alvarez RA, Acland GM, Aguirre GD. Plasma lipid abnormalities in the miniature poodle with progressive rod-cone degeneration. Exp Eye Res 1991; 52 : 349-355.

45. Anderson RE, Maude MB, Nilsson SE, Narfstrom K. Plasma lipid abnormalities in the abyssinian cat with a hereditary rod-cone degeneration. Exp Eye Res 1991; 53: 415-417.

46. Connor WE, Weleber RG, DeFrancesco C, Lin DS, Wolf DP. Sperm abnormalities in retinitis pigmentosa. Invest Ophthalmol Vis Sci 1997; 38: 2619-2628.

47. Correa-Cerro LS, Wassif CA, Kratz L, Miller GF, Munasinghe JP, Grinberg A et al. Development and characterization of a hypomorphic Smith-Lemli-Opitz syndrome mouse model and efficacy of simvastatin therapy. Hum Mol Genet 2006; 15: 839-851.

48. Maxfield FR, Tabas I. Role of cholesterol and lipid organization in disease. Nature 2005 438: 612-621.

49. Vaughan DK, Peachey NS, Richards MJ, Buchan B, Fliesler SJ. Light-induced exacerbation of retinal degeneration in a rat model of Smith-Lemli-Opitz syndrome. Exp Eye Res 2006; 82: 496-504.

50. Bazan NG, Scott BL, Reddy TS, Pelias MZ. Decreased content of docosahexaenoate and arachidonate in plasma phospholipids in Usher's syndrome. Biochem Biophys Res Commun 1986; 141: 600-604.

51. Delton-Vandenbroucke I, Maude MB, Chen H, Aguirre GD, Acland GM, Anderson RE. Effect of diet on the fatty acid and molecular species composition of dog retina phospholipids. Lipids 1998; 33: 1187-1193.

52. Gong J, Rosner B, Rees DG, Berson EL, Weigel-DiFranco CA, Schaefer EJ. Plasma docosahexaenoic acid levels in various genetic forms of retinitis pigmentosa. Invest Ophthalmol Vis Sci 1992; 33: 2596-2602.

53. Hoffman DR, DeMar JC, Heird WC, Birch DG, Anderson RE. Impaired synthesis of DHA in patients with X-linked retinitis pigmentosa. J Lipid Res 2001; 42: 1395-1401.

54. Maude MB, Anderson EO, Anderson RE. Polyunsaturated fatty acids are lower in blood lipids of Usher's type I but not Usher's type II. Invest Ophthalmol Vis Sci 1998; 39: 2164-2166.

55. Simonelli F, Manna C, Romano N, Nunziata G, Voto O, Rinaldi E. Evaluation of fatty acids in membrane phospholipids of erythrocytes in retinitis pigmentosa patients. Ophthalmic Res 1996; 28: 93-98.

56. Richards MJ, Nagel BA, Fliesler SJ. Lipid hydroperoxide formation in the retina: correlation with retinal degeneration and light damage in a rat model of Smith-Lemli-Opitz syndrome. Exp Eye Res 2006; 82: 538-541.
57. Artwohl M, Roden M, WaldhausI W, Freudenthaler A, Baumgartner-Parzer SM. Free fatty acids trigger apoptosis and inhibit cell cycle progression in human vascular endothelial cells. Faseb J 2004; 18: 146-148.

58. Shimabukuro M, Zhou YT, Levi M, Unger RH. Fatty acid-induced beta cell apoptosis: a link between obesity and diabetes. Proc Natl Acad Sci USA 1998; 95: 2498-2502.

59. Leung HT, Tseng-Crank J, Kim E, Mahapatra C, Shino S, Zhou Y et al. DAG lipase activity is necessary for TRP channel regulation in Drosophila photoreceptors. Neuron 2008; 58 : 884-896.

60. Das UN. Essential fatty acids, lipid peroxidation and apoptosis. Prostaglandins Leukot Essent Fatty Acids 1999; 61: 157-163.

61. Johnson RA, Hamilton JA, Worgall TS, Deckelbaum RJ. Free fatty acids modulate intermembrane trafficking of cholesterol by increasing lipid mobilities: novel 13C NMR analyses of free cholesterol partitioning. Biochemistry 2003; 42: 1637-1645

62. Aikens J, Dix TA. Perhydroxyl radical (HOO.) initiated lipid peroxidation. The role of fatty acid hydroperoxides. J Biol Chem 1991; 266: 15091-15098.

63. Spiteller G. Are changes of the cell membrane structure causally involved in the aging process? Ann N Y Acad Sci 2002; 959: 30-44.

64. Chandra J, Samali A, Orrenius S. Triggering and modulation of apoptosis by oxidative stress. Free Radic Biol Med 2000; 29: 323-333.

65. Kim JS, He L, Lemasters JJ. Mitochondrial permeability transition: a common pathway to necrosis and apoptosis. Biochem Biophys Res Commun 2003; 304: 463-470.

66. Greenberg ME, Li XM, Gugiu BG, Gu X, Qin J, Salomon RG et al. The lipid whisker model of the structure of oxidized cell membranes. J Biol Chem 2008; 283: 2385-2396.

67. Organisciak DT, Darrow RM, Jiang YL, Blanks JC. Retinal light damage in rats with altered levels of rod outer segment docosahexaenoate. Invest Ophthalmol Vis Sci 1996; 37: 2243-2257.

68. Bloomquist BT, Shortridge RD, Schneuwly S, Perdew M, Montell C, Steller H et al. Isolation of a putative phospholipase $\mathrm{C}$ gene of Drosophila, norpA, and its role in phototransduction. Cell 1988; 54: 723-733.

69. Ferreira PA, Pak WL. Bovine phospholipase $C$ highly homologous to the norpA protein of Drosophila is expressed specifically in cones. J Biol Chem 1994; 269: 3129-3131.

70. Ferreira PA, Shortridge RD, Pak WL. Distinctive subtypes of bovine phospholipase $C$ that have preferential expression in the retina and high homology to the norpA gene product of Drosophila. Proc Natl Acad Sci USA 1993; 90: 6042-6046.

71. Ghalayini A, Anderson RE. Phosphatidylinositol 4,5-bisphosphate: light-mediated breakdown in the vertebrate retina. Biochem Biophys Res Commun 1984; 124: 503-506.

72. Ghalayini AJ, Tarver AP, Mackin WM, Koutz CA, Anderson RE. Identification and immunolocalization of phospholipase C in bovine rod outer segments. J Neurochem 1991; 57: 1405-1412

73. Choe HG, Ghalayini AJ, Anderson RE. Phosphoinositide metabolism in frog rod outer segments. Exp Eye Res 1990; 51: 167-176.

74. Hayashi F, Amakawa T. Light-mediated breakdown of phosphatidylinositol-4,5bisphosphate in isolated rod outer segments of frog photoreceptor. Biochem Biophys Res Commun 1985; 128: 954-959.

75. Hayashi F, Sumi M, Amakawa T. Phosphatidylinositol stimulates phosphorylation of protein components I and II in rod outer segments of frog photoreceptors. Biochem Biophys Res Commun 1987; 148: 54-60.

76. Millar FA, Fisher SC, Muir CA, Edwards E, Hawthorne JN. Polyphosphoinositide hydrolysis in response to light stimulation of rat and chick retina and retinal rod outer segments. Biochim Biophys Acta 1988; 970: 205-211.

77. Gehm BD, Mc Connell DG. Phosphatidylinositol-4,5-bisphosphate phospholipase C in bovine rod outer segments. Biochemistry 1990; 29: 5447-5452.

78. Gehm BD, Pinke RM, Laquerre S, Chafouleas JG, Schultz DA, Pepperl DJ et al. Activation of bovine rod outer segment phosphatidylinositol-4,5-bisphosphate phospholipase $C$ by calmodulin antagonists does not depend on calmodulin. Biochemistry 1991; 30: 11302-11306.

79. Peng YW, Rhee SG, Yu WP, Ho YK, Schoen T, Chader GJ et al. Identification of components of a phosphoinositide signaling pathway in retinal rod outer segments. Proc Natl Acad Sci USA 1997; 94: 1995-2000.

80. Neilson DE, Eiben RM, Waniewski S, Hoppel CL, Varnes ME, Bangert BA et al. Autosomal dominant acute necrotizing encephalopathy. Neurology 2003; 61: 226-230.

81. Neilson DE, Feiler HS, Wilhelmsen KC, Lynn A, Eiben RM, Kerr DS et al. Autosomal dominant acute necrotizing encephalopathy maps to 2q12.1-2q13. Ann Neurol 2004; 55: 291-294.

82. Krebber $\mathrm{H}$, Bastians $\mathrm{H}$, Hoheisel J, Lichter $\mathrm{P}$, Ponstingl $\mathrm{H}$, Joos $\mathrm{S}$. Localization of the gene encoding the Ran-binding protein RanBP2 to human chromosome $2 q 11-q 13$ by fluorescence in situ hybridization. Genomics 1997; 43: 247-248.

83. Ferreira PA. Characterization of RanBP2-associated molecular components in neuroretina. Methods enzymol 2000; 315: 455-468. 